\title{
Enhancing Transparency of a Position-Exchange Teleoperator
}

\author{
Mohsen Mahvash and Allison M. Okamura \\ Department of Mechanical Engineering \\ Engineering Research Center for Computer-Integrated Surgical Systems and Technology \\ The Johns Hopkins University, USA \\ E-mail: \{mahvash, aokamura\}@jhu.edu
}

\begin{abstract}
Dynamic properties of robotic manipulators, including inertia, damping, and friction, limit the transparency of a haptic-feedback teleoperator. In this paper, we develop a position-exchange controller to provide hapticfeedback for a surgical teleoperator. The controller consists of proportional controllers and model-based feedforward terms that cancel the dynamic properties of the manipulators. We show that the teleoperator transmits the impedance of a soft environment to the operator when the gains of the proportional controllers are very high and dynamic terms of the manipulators are canceled. However, the high gains and complete cancellation of the dynamic terms of the manipulators can make the teleoperator unstable. We use Llewellyn's criteria for absolute stability to limit the controller parameters to values that keep the teleoperator stable during interactions with any passive user and environment. Experimental results using a custom version of the da Vinci Surgical System show that $70 \%$ of the inertia of the slave manipulators during low-frequency motion and almost all static friction of the manipulators during sliding motion can be canceled.
\end{abstract}

\section{Introduction}

A haptic-feedback teleoperator consists of a master manipulator, a slave manipulator, and a controller that virtually connects the manipulators. Dynamic properties of the manipulators of a non-delayed hapticfeedback teleoperator, including inertia, damping, and friction, limit the performance of the teleoperator. For fast motions, the inertia of the manipulators affects the performance of the operator in performing tasks that involve frequent changes of motion direction. The inertia masks the forces transmitted to the operator from the environment, causes operator fatigue, and reduces the control of the operator to position the manipulators. For slow motions, friction dominates.

Transparency is the most common measure to evaluate the performance of a haptic-feedback teleoperator. An ideal transparent teleoperator transmits the exact forces of the teleoperated environment to the operator and the slave manipulators should exactly follow the position of the master manipulator $[1,2]$. Consequently, an ideal transparent teleoperator should transmit the same impedance of the teleoperated environment to the operator.

Transparency of a haptic-feedback teleoperator depends on its control architecture type, which is defined by how force and position signals are exchanged between the master and slave manipulators. The most common control architectures are the position-position (or position-exchange), position-force, force-position, force-force, and the four-channel control architecture introduced by Lawrence [1]. The four-channel control architecture provides ideal transparency for an ideal non-delayed teleoperator [1]. None of the other control architectures provides ideal transparency, even for an ideal teleoperator.

In this paper, our motivation is to develop haptic feedback for a surgical teleoperator. The use of force sensors is significantly limited in surgical teleoperators, due to practical considerations [3]. Therefore, we use a position-exchange teleoperator that does not require any force sensor.

A position-exchange controller consists of two proportional controllers that cause the master and slave manipulators track the positions of each other and two feedforward model-based terms that cancel the dynamic properties of the manipulators. A positionexchange teleoperator does not provide ideal transparency, but we show that it transmits the impedance of a soft environment to the operator when the gains of 
the proportional controllers are very high and dynamic terms of the manipulators are cancelled.

Perfect cancelation of the dynamic terms of the manipulators is not possible due to non-idealities of teleoperators, including encoder noise, dynamics of tendon-driven joints, and model uncertainties. We use Llewellyn's criteria for absolute stability to define the parameters of the controllers such that they preserve the stability of the teleoperator. Experimental results performed on a da Vinci surgical system show cancellation of about $70 \%$ of the inertia of the slave manipulators in low frequencies and almost all the static friction during sliding motion.

\section{Related Work}

Different methods have been recently presented to increase the transparency of a position-exchange teleoperator while its stability is preserved. HashtrudiZaad and Salcudean [4] discussed the use of absolute stability theory [5] for designing position-tracking controllers for a teleoperator. $\mathrm{Ni}$ and Wang [6] used a gain-switching method to increase transparency of a position-exchange teleoperator. Our recent work used model-based friction compensators to increase transparency of a position-exchange teleoperator with compliant tendon-driven joints $[7,8]$.

\section{Position-Exchange Controllers}

In order to clarify the analysis of a teleoperator, we consider one degree-of-freedom (DOF) linear manipulators. In general, the behavior of a teleoperator designed for impedance control is smooth enough to be modeled by a linear model in the neighborhood of an operating point. The model for the master and slave manipulators are written as:

$$
\begin{aligned}
m_{m} \ddot{x}_{m}+b_{m} \dot{x}_{m}+g_{m}+f_{f m} & =f_{c m}+f_{h} \\
m_{s} \ddot{x}_{s}+b_{s} \dot{x}_{s}+g_{s}+f_{f s} & =f_{c s}-f_{e}
\end{aligned}
$$

respectively. $x$ is the position of the manipulator, $m$ is the mass, $b$ is the damping coefficient, $g$ is the gravity term, $f_{f}$ is the Coulomb friction force, $f_{h}$ is the user force, $f_{e}$ is the environment force, and $f_{c}$ is the controller force. The subscripts $m$ and $s$ refer to the master and slave, respectively.

Control objectives for a transparent haptic teleoperator are [2]: position tracking, $x_{m}=x_{s}$, and force tracking, $f_{h}=f_{e}$. We used position-exchange controllers to meet the above objectives. The controllers include feedforward terms that compensate for the friction, gravity, damping, and inertia of the manipulators

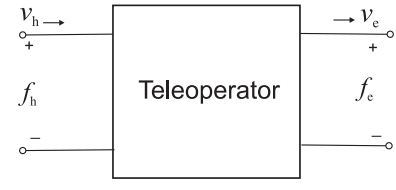

\section{Figure 1. Two-port representation of a teleop- erator}

and proportional position feedback that provides position tracking and consequently haptic feedback:

$$
\begin{array}{r}
f_{c m}=\hat{f}_{f m}+\hat{g}_{m}+m_{m}^{\prime} \ddot{x}_{s}^{f}+b_{m}^{\prime} \dot{x}_{m}^{f}+k\left(x_{m}-x_{s}\right) \\
f_{c s}=\hat{f}_{f s}+\hat{g}_{s}+m_{s}^{\prime} \ddot{x}_{m}^{f}+b_{s}^{\prime} \dot{x}_{m}^{f}+k\left(x_{s}-x_{m}\right)
\end{array}
$$

where $\ddot{x}_{m}^{f}, \dot{x}_{s}^{f}, \ddot{x}_{s}^{f}$, and $\dot{x}_{m}^{f}$ are low-pass-filtered accelerations and velocities of the master and slave manipulators, $m_{m}^{\prime}$ and $m_{s}^{\prime}$ are mass coefficients, $b_{m}^{\prime}$ and $b_{s}^{\prime}$ are damping coefficients, $\hat{g}_{m}$ and $\hat{g}_{s}$ are gravity terms, and $\hat{f}_{f m}$ and $\hat{f}_{f s}$ are friction forces of the controllers. The mass and damping coefficients of the controllers estimates the actual coefficients of the manipulators. These coefficients must be scaled down to preserve the absolute stability of the teleoperator, as discussed in the following sections.

The friction forces $\hat{f}_{f m}$ and $\hat{f}_{f s}$ are generated by model-based friction compensators that use the singleelastic friction model or the Dahl model to estimate friction forces of the manipulators. We showed in [8] that these compensators accurately estimate friction in a tendon-driven joint (or a direct-driven joint) during sliding motion of the joint and preserve the passivity of the joint. From now, we remove friction forces and gravity forces from the model to clarify the analysis.

Velocity and acceleration terms of controllers are calculated by the first and second derivatives of the positions of the manipulators and then are filtered by first and second order low-pass filters to remove encoder noise:

$$
\frac{1}{\left(1+\frac{s}{\omega_{0}}\right)} \text { and } \frac{1}{\left(1+\frac{s}{\omega_{0}}\right)^{2}},
$$

where $\omega_{0}$ is the cut off frequency of the filters. These filters might also prevent high-frequency unmodeled dynamics due to transmission cables of the manipulators being activated.

\section{Two-Port Linear Representation}

We use the two-port linear representation to study the stability and the transparency of a teleoperator [9]. 
Figure 1 shows a two-port representation for a teleoperator where inputs (flows) are: $v_{e}=v_{s}=\dot{x}_{s}$ and $v_{h}=v_{m}=\dot{x}_{m}$ and outputs (efforts) are $f_{h}$ and $f_{e}$. The dynamic equations of the controlled teleoperator are obtained from (1) and (2) as

$$
\begin{gathered}
m_{m} \ddot{x}_{m}+b_{m} \dot{x}_{m}+k x_{m}-\left(m_{m}^{\prime} \ddot{x}_{s}^{f}+b_{m}^{\prime} \dot{x}_{m}^{f}+k x_{s}\right) \\
=f_{h}+\hat{f}_{f m}-f_{f m}+\hat{g}_{m}-g_{m} \approx f_{h} \\
m_{s} \ddot{x}_{s}+b_{s} \dot{x}_{s}+k x_{s}-\left(m_{s}^{\prime} \ddot{x}_{m}^{f}+b_{s}^{\prime} \dot{x}_{m}^{f}+k x_{m}\right) \\
=-f_{e}+\hat{f}_{f s}-f_{f s}+\hat{g}_{s}-g_{s} \approx-f_{e}
\end{gathered}
$$

The impedance form for a two-port network is defined as

$$
\left[\begin{array}{l}
F_{h} \\
F_{e}
\end{array}\right]=\left[\begin{array}{ll}
z_{11} & z_{12} \\
z_{21} & z_{22}
\end{array}\right]\left[\begin{array}{c}
V_{m} \\
-V_{s}
\end{array}\right]
$$

where $V_{m}, V_{s}, F_{h}$, and $F_{e}$ are Laplace transforms of $v_{m}$, $v_{s}, f_{h}$, and $f_{e}$, respectively. Taking Laplace transform of (4) and combining it with (5), the impedance matrix for the controlled teleoperator is obtained as

$$
\begin{aligned}
z_{11} & =m_{m} s+b_{m}+\frac{k}{s} \\
z_{12} & =\frac{m_{m}^{\prime} s}{\left(1+\frac{s}{\omega_{0}}\right)^{2}}+\frac{b_{m}^{\prime}}{1+\frac{s}{\omega_{0}}}+\frac{k}{s} \\
z_{21} & =\frac{m_{s}^{\prime} s}{\left(1+\frac{s}{\omega_{0}}\right)^{2}}+\frac{b_{s}^{\prime}}{1+\frac{s}{\omega_{0}}}+\frac{k}{s} \\
z_{22} & =m_{s} s+b_{s}+\frac{k}{s}
\end{aligned}
$$

\section{Teleoperator Performance}

The way we evaluate the performance of a teleoperator is to compare the impedance of the environment with the impedance transmitted to the user. Considering the impedance matrix $(5), z_{t}$ the impedance transmitted to the user, is obtained by

$$
z_{t}=\frac{F_{h}}{V_{m}}=z_{11}-\frac{z_{12} z_{21}}{z_{22}+z_{e}}
$$

where $z_{e}$ is the impedance of the environment, such that $F_{e}=z_{e} V_{s}$.

Lawrence [1] showed that a position-exchange teleoperator does not provide impedance match at the extremes of $z_{e}$. Using (7), we write

$$
\begin{aligned}
\lim _{z_{e} \rightarrow \infty} z_{t} & =z_{11} \\
\lim _{z_{e} \rightarrow 0} z_{t} & =z_{11}-\frac{z_{12} z_{21}}{z_{22}}
\end{aligned}
$$

which confirm there is no impedance match at the extremes of $z_{e}$.
Using (7), we can also write

$$
z_{t}=\frac{z_{11}}{z_{22}+z_{e}} z_{e}+\frac{z_{11} z_{22}-z_{12} z_{21}}{z_{22}+z_{e}}
$$

Substituting the values of (6) into (9), we obtain

$$
\begin{array}{r}
z_{t}=\frac{m_{m} s+b_{m}+\frac{k}{s}}{m_{s} s+b_{s}+\frac{k}{s}+z_{e}} z_{e}+ \\
\frac{\left(m_{m} s+b_{m}+\frac{k}{s}\right)\left(m_{s} s+b_{s}+\frac{k}{s}\right)}{m_{s} s+b_{s}+\frac{k}{s}+z_{e}}- \\
\frac{\left(\frac{m_{m}^{\prime} s}{\left(1+\frac{s}{\omega_{0}}\right)^{2}}+\frac{b_{m}^{\prime}}{\left(1+\frac{s}{\omega_{0}}\right)}+\frac{k}{s}\right)\left(\frac{m_{s}^{\prime} s}{\left(1+\frac{s}{\omega_{0}}\right)^{2}}+\frac{b_{s}^{\prime}}{\left(1+\frac{s}{\omega_{0}}\right)}+\frac{k}{s}\right)}{m_{s} s+b_{s}+\frac{k}{s}+z_{e}} .
\end{array}
$$

For $\frac{k}{s} \gg 1$,

$$
\begin{array}{r}
z_{t}=z_{e}+m_{m} s+b_{m}+m_{s} s+b_{s} \\
-\frac{m_{m}^{\prime} s}{\left(1+\frac{s}{\omega_{0}}\right)^{2}}-\frac{b_{m}^{\prime}}{1+\frac{s}{\omega_{0}}}-\frac{m_{s}^{\prime} s}{\left(1+\frac{s}{\omega_{0}}\right)^{2}}-\frac{b_{s}^{\prime}}{1+\frac{s}{\omega_{0}}}
\end{array}
$$

This shows that, for high gains and small $z_{e}$, a positionexchange teleoperator at low frequencies transmits the impedance of a soft environment to the operator when the impedances of the master and slave manipulators are fully compensated. This is applicable to surgical teleoperators where the environment is soft and the user operates at low frequencies.

\section{Absolute Stability}

We use absolute stability (or unconditional stability) theory to study the stability of a teleoperator. A two-port network is absolutely stable when it remains stable under all possible uncoupled passive inputs at its ports [5].

The necessary and sufficient conditions for absolute stability of a linear two-port network (Llewellyn's criteria) based on its impedance matrix are [5]:

1. $z_{11}(s)$ and $z_{22}(s)$ have no poles in the right half plane.

2. Any poles of $z_{11}(s)$ and $z_{22}(s)$ on the imaginary axis are simple with real and positive residues, and

3. For all real values of $\omega$, we have

$$
\begin{array}{cc}
\text { a) } & \operatorname{Re}\left(z_{11}\right) \geq 0 \\
\text { b) } & \operatorname{Re}\left(z_{22}\right) \geq 0 \\
\text { c) } & 2 \operatorname{Re}\left(z_{11}\right) \operatorname{Re}\left(z_{22}\right) \geq \operatorname{Re}\left(z_{12} z_{21}\right)+\left|z_{12} z_{21}\right|
\end{array}
$$

Conditions 1, 2, 3a, and 3b only depend on the dynamics of the manipulators and the proportional gain 
of the controllers. These conditions are always satisfied for the impedance terms in (6). The condition 3c for the impedance terms in (6) is written as

$$
2 b_{m} b_{s} \geq \operatorname{Re}\left(z_{12} z_{21}\right)+\left|z_{12} z_{21}\right|
$$

We set

$$
b_{s}^{\prime}=0, b_{m}^{\prime}=0
$$

to increase the stability margin of the controller. We also consider the stability analysis for a teleoperator that its master manipulator has small mass. This is a reasonable assumption for a manipulator designed as a haptic device. Therefore, we choose

$$
m_{m}^{\prime}=0
$$

It is shown in the Appendix that (11) is satisfied if

$$
\begin{aligned}
k & \geq 0.125 \omega_{0}^{2} m_{s}^{\prime} \\
b_{m} b_{s} & \geq 1.3 k m_{s}^{\prime} .
\end{aligned}
$$

Combining the above conditions concludes

$$
7.0 \sqrt{b_{m} b_{s}} \geq \omega_{0} m_{s}^{\prime}
$$

We use the following rules to calculate the parameters of the controllers that provide maximum transparency and stability.

- $m_{s}^{\prime}$ should be the closest value to $m_{s}$ that satisfies (15)

- $\omega_{0}$ should be the highest frequency that satisfies (15) and prevents quantization noise of encoders causing oscillations and unmodeled dynamics due to transmission cables being activated.

- $k$ should be the biggest gain that satisfies (14) and prevents quantization noise from causing oscillations and unmodeled dynamics being activated.

\section{$7 \quad$ Experimental Results}

The position-exchange controller of this work was implemented to experimentally evaluate its performance. The experiments were conducted on a custom version of da Vinci telerobotic system [10, 11] (Figure 2). The telerobotic system contains two master manipulators and two slave manipulators provided by Intuitive Surgical, Inc., and a custom control system developed at the Johns Hopkins University. There is significant Coulomb friction and inertia in the da Vinci slave manipulator and no local force control loop can be used to scaled them down [8]. The hardware of the control system includes motor controllers connected to a personal computer [12]. The control system program consisted of a $1 \mathrm{kHz}$ real-time process running under Real Time Application Interface (RTAI) for Linux and a user-interface process.

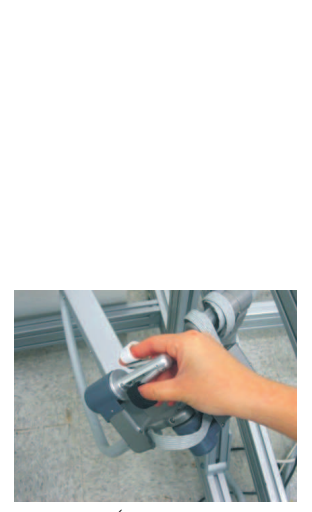

(a)

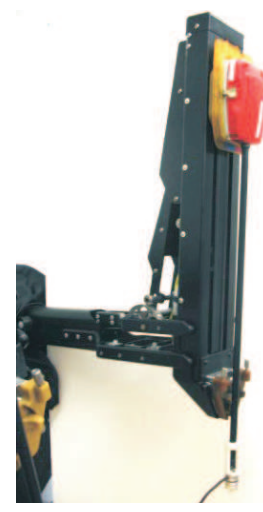

(b)
Figure 2. The custom da Vinci surgical system: (a) master telemanipulator (MTM) and (b) patient-side manipulator (PSM).

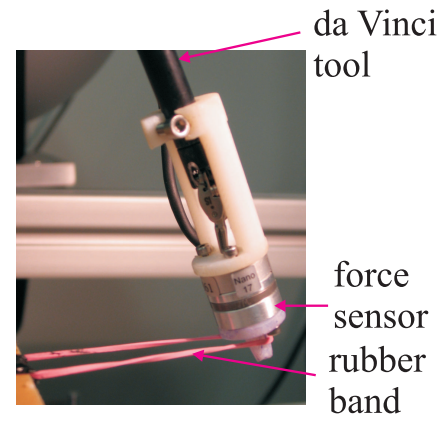

Figure 3. Setup for measuring force responses of contact with a soft object.

\subsection{Controllers}

The custom control system implements proportional tracking controllers in Cartesian space, Dahl-based friction compensators [8], and inertia compensators in joint space of the slave manipulators. We ignore possible couplings between the degrees of freedom of the teleoperator. The teleoperator equations for each joint of the slave manipulator were written in the format of model (1). Then design rules of Section 6 were used to obtain control parameters for each joint. We were able to implement an absolutely stable position-exchange controller for each joint with following parameters $\omega_{0}$ $=12 \mathrm{~Hz}, k=10 \mathrm{~N} / \mathrm{cm}, m_{s}^{\prime} \approx .75 m_{s}$, and $f_{D}=f_{C}$ where $f_{D}$ and $f_{C}$ are Coulomb friction levels of the compensator and the joint, respectively [7]. 


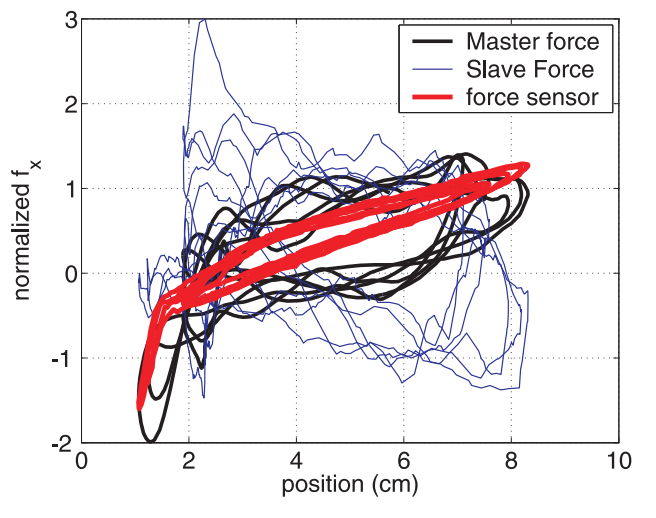

Figure 4. Force-displacement curves along the $x$ axis during stretching of a rubber band for several cycles when friction and inertia of the slave manipulator are compensated.

\subsection{Performance Comparison}

Two sets of experiments were performed to show the effect of friction and inertia compensation on performance of the teleoperator: contact with an elastic environment, and free space movement.

\subsubsection{Contacting an Elastic Environment}

Here, we compare the force responses of the da Vinci system when the slave manipulator stretched a rubber band several times. An ATI Nano-17 force sensor attached to the tip of the slave manipulator (Figure 3 ) directly measured the force responses of stretching the band. The force from the sensor was only used to evaluate the compensation method, and was not used by the controller. The forces applied by the actuators are estimated by the current applied to the actuator. As the force-displacement curves in Figure 4 show, friction and inertia compensation increases the match between force-displacement curves of the master and the environment. Thus, the match between the impedance of the environment and the transmitted impedance to the user is improved when inertia and friction compensation are used. The reported forces are normalized to the total Coulomb friction level of the prismatic joint in order to protect proprietary data of Intuitive Surgical, Inc.

\subsubsection{Free Space Movement}

Forces applied to the operator during free space movement cause operator's fatigue. Figure 5 compares the

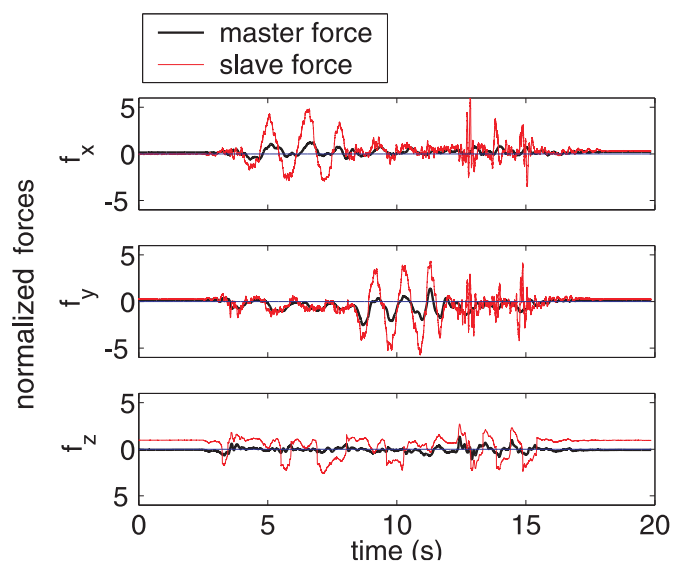

Figure 5. Force-time responses of the master and slave manipulators when the slave manipulator moves in free space and friction and inertia compensation are applied.

force responses applied to the tip of the slave and master manipulators by the controller during inertia and friction compensation when the slave manipulator moves in free space. Friction and inertia compensation reduce the force applied to the master manipulator and consequently the user.

\section{Conclusions}

A haptic-feedback teleoperator with a positionexchange control structure was developed. The control structure contained high-gain proportional controllers and model-based compensators to provide position tracking and a close match between the impedance of the soft environment and the impedance transmitted to the user. The control structure is appropriate for having haptic feedback for surgical teleoperators, where the use of force sensors is limited and the teleoperated environment is soft. It was shown analytically and experimentally that the controllers preserved the absolute stability of the teleoperator and enhanced its transparency in transmitting the impedance of a soft environment to the user. Future work includes studying the effect of unmodeled dynamics, including tendon dynamics and coupling between degrees of freedoms in the absolute stability of a teleoperator.

\section{Acknowledgments}

This work supported in part by National Science Foundation Grant No. EIA-0312551, National In- 
stitutes of Health Grant No. R01-EB002004, and Whitaker Foundation Grant No. RG-02-911. We would also like to acknowledge the support of Intuitive Surgical Inc., Sunnyvale, CA, USA. The authors thank Ankur Kapoor and Dr. Peter Kazanzides for their contributions in designing the electronic hardware.

\section{Appendix}

It is shown that (11) holds if

$$
\begin{aligned}
k & \geq 0.125 \omega_{0}^{2} m_{s}^{\prime} \\
b_{m} b_{s} & \geq 1.3 k m_{s}^{\prime} .
\end{aligned}
$$

Based on the triangle inequality,

$$
\left|\operatorname{Im}\left(z_{12} z_{21}\right)\right|+\left|\operatorname{Re}\left(z_{12} z_{21}\right)\right| \geq\left|z_{12} z_{21}\right| .
$$

Considering (17), (11) is satisfied, if

$$
2 b_{m} b_{s} \geq \operatorname{Re}\left(z_{12} z_{21}\right)+\left|\operatorname{Im}\left(z_{12} z_{21}\right)\right|+\left|\operatorname{Re}\left(z_{12} z_{21}\right)\right| \text {. }
$$

If

$$
\operatorname{Re}\left(z_{12} z_{21}\right) \leq 0
$$

then (18) can be written as

$$
2 b_{m} b_{s} \geq\left|\operatorname{Im}\left(z_{12} z_{21}\right)\right|
$$

Considering (6), (12), and (13), we write

$$
\begin{aligned}
\left(z_{12} z_{21}\right)_{s=j \omega} & =\left(\frac{k m_{s}^{\prime}}{\left(1+\frac{s}{\omega_{0}}\right)^{2}}+\frac{k^{2}}{s^{2}}\right)_{s=j \omega} \\
& =\frac{k^{2}}{\omega^{2}}\left(\frac{\frac{m_{s}^{\prime}}{k}\left(1-\left(\frac{\omega}{\omega_{0}}\right)^{2}\right) \omega^{2}}{\left(1+\left(\frac{\omega}{\omega_{0}}\right)^{2}\right)^{2}}-1\right) \\
& -j \frac{2 k m_{s}^{\prime} \frac{\omega}{\omega_{0}}}{\left(1+\left(\frac{\omega}{\omega_{0}}\right)^{2}\right)^{2}}
\end{aligned}
$$

We plotted selected terms of $\operatorname{Re}\left(z_{12} z_{21}\right)$ and $\left|\operatorname{Im}\left(z_{12} z_{21}\right)\right|$ verses $\frac{\omega}{\omega_{0}}$ and concluded that for any $\omega \geq 0$,

$$
\begin{gathered}
\operatorname{Re}\left(z_{12} z_{21}\right)=\frac{k^{2}}{\omega^{2}}\left(\frac{\frac{m_{s}^{\prime}}{k}\left(1-\left(\frac{\omega}{\omega_{0}}\right)^{2}\right) \omega^{2}}{\left(1+\left(\frac{\omega}{\omega_{0}}\right)^{2}\right)^{2}}-1\right) \\
\leq \frac{k^{2}}{\omega^{2}}\left(0.125 \frac{m_{s}^{\prime} \omega_{0}^{2}}{k}-1\right), \text { and } \\
\left|\operatorname{Im}\left(z_{12} z_{21}\right)\right|=\frac{2 k m_{s}^{\prime} \frac{\omega}{\omega_{0}}}{\left(1+\left(\frac{\omega}{\omega_{0}}\right)^{2}\right)^{2}} \leq 1.3 k m_{s}^{\prime}
\end{gathered}
$$

Considering (21) and (22), (19) and (20) are satisfied if

$$
\begin{gathered}
k \geq 0.125 \omega_{0}^{2} m_{s}^{\prime} \\
b_{m} b_{s} \geq 1.3 k m_{s}^{\prime}
\end{gathered}
$$

\section{References}

[1] D. A. Lawrence, "Stability and transparency in bilateral teleoperation," IEEE Transactions on Robotics and Automation, vol. 9, no. 5, pp. 624-637, 1993.

[2] Y. Yokokohji and T. Yoshikawa, "Bilateral control of master-slave manipulators for ideal kinesthetic coupling-formulation and experiment," IEEE Transactions on Robotics and Automation, vol. 10, no. 5, pp. 605-620, 1994.

[3] A. M. Okamura, "Methods for haptic feedback in teleoperated robot-assisted surgery," Industrial Robot, vol. 31, no. 6, pp. 499-508, 2004.

[4] K. Hashtrudi-Zaad and S. E. Salcudean, "Analysis of control architectures for teleoperation systems with impedance/admittance master and slave manipulators," International Journal of Robotics Research, vol. 20, no. 6, pp. 419-445, 2001.

[5] S. S. Haykin, Active Network Theory. AddisonWesley, 1970.

[6] L. Ni and D. Wang, "A gain-switching control scheme for position-error-based bilateral teleoperation: Contact stability analysis and controller design," International Journal of Robotics Research, vol. 23, no. 3, pp. 555-574, 2004.

[7] M. Mahvash and A. Okamura, "Friction compensation for a force-feedback telerobotic system," in IEEE International Conference on Robotics and Automation, Orlando, Florida, 2006, pp. 3268-3273.

[8] — , "Friction compensation for a force-feedback teleoperator with compliant transmission," in 45th IEEE Conf. on Decision and Control, San Diego, CA, 2006, pp. $4508-4513$.

[9] B. Hannaford, "A design framework for teleoperators with kinesthetic feedback," IEEE Transactions on Robotics and Automation, vol. 5, pp. 426-434, 1989.

[10] Intutive Surgical Inc. http://www.intuitivesurgical.com/.

[11] G. S. Guthart and J. K. Salisbury, "The Intuitive telesurgery system: Overview and application," in Proc. IEEE Int. Conf. Robotics and Automation, San Francisco, CA, April 2000, pp. 618-621.

[12] A. Kapoor, N. Simaan, and P. Kazanzides, "A system for speed and torque control of DC motors with application to small snake robots," in IEEE/APS Conf. Mechatron. and Robot, 2004. 